\title{
Is total arch replacement associated with an increased risk after acute type A dissection?
}

\author{
Mohamed Salem $^{1 \#}$, Christine Friedrich ${ }^{1 \#}$, Rene Rusch ${ }^{1}$, Derk Frank ${ }^{2}$, Grischa Hoffmann ${ }^{1}$, Georg Lutter ${ }^{1}$, \\ Rouven Berndt ${ }^{1}$, Jochen Cremer ${ }^{1}$, Assad Haneya ${ }^{1}$, Thomas Puehler ${ }^{1}$ \\ ${ }^{1}$ Department of Cardiac and Vascular Surgery, University Medical Center Schleswig-Holstein, Campus Kiel, Kiel, Germany; ${ }^{2}$ Department of \\ Cardiology and Angiology, University Medical Center Schleswig-Holstein, Campus Kiel, Kiel, Germany \\ Contributions: (I) Conception and design: M Salem, T Puehler; (II) Administrative support: M Salem, C Friedrich, J Cremer, A Haneya, R Rusch, R \\ Berndt, T Puehler, D Frank; (III) Provision of study materials or patients: M Salem, J Cremer, A Haneya, T Puehler, D Frank; (IV) Collection and \\ assembly of data: M Salem, C Friedrich; (V) Data analysis and interpretation: M Salem, C Friedrich; (VI) Manuscript writing: All authors; (VII) Final \\ approval of manuscript: All authors. \\ \#These authors contributed equally to this work. \\ Correspondence to: Mohamed Salem, MD. Department of Cardiac and Vascular Surgery, University Medical Center Schleswig-Holstein, Campus Kiel, \\ Arnold-Heller Strasse 3, D-24105 Kiel, Germany. Email: mohamed.salem@uksh.de.
}

Background: The surgical strategy for acute type A aortic dissection (AADA) usually consists of reconstruction of the tear-lesion in the affected part of the ascending aorta. The optimal strategy either to replace the ascending aorta (AAR) or to replace the ascending aorta and the total aortic arch (TAAR) is still under debate. Our study compares the 30-day mortality between AAR and TAAR in AADA surgery.

Methods: In this retrospective observational study, we analysed a total patient cohort of 339 patients who underwent surgery for AADA from January 2001 until December 2016. A propensity score-matched analysis between the AAR- and the TAAR-group with 43 patients for each subgroup was subsequently carried out. A multivariable analysis was performed to identify risk-factors for the 30-d-mortality. The 30-day mortality was defined as the primary end-point and long-term survival was the secondary endpoint.

Results: In 292 (86.1\%) patients AAR and in 47 (13.9\%) patients TAAR was performed for emergent AADA. Patients were older $(\mathrm{P}=0.049)$ in the AAR group. The median log Euro-SCORE was $25.5 \%$ $(12.7 ; 41.7)$ for AAR and $19.7 \%(11.7 ; 32.2)$ for the TAAR patient cohort $(\mathrm{P}=0.12)$. Operative time, cardiopulmonary bypass- $(\mathrm{CPB})$, cross-clamp- and ischemic time were significantly longer in the TAAR group $(\mathrm{P}<0.001)$. The overall 30-day mortality-rate was $17.7 \%(\mathrm{n}=60)$ but was not significantly different between the two groups $(\mathrm{P}=0.27)$. Forty-nine $(16.8 \%)$ patients died in the AAR and 11 patients $(23.4 \%)$ in the TAAR group. After propensity-score matching, no difference in mortality was seen between the subgroups as well $(\mathrm{P}=0.44)$. Multivariable analysis identified the Euro-SCORE, long operation-time, postoperative dialysis and arrhythmia and administration of red blood cell concentrates as risk factors for 30-day mortality, but not for TAAR versus AAR.

Conclusions: The therapeutic goal in AADA surgery should be the complete restoration of the aorta to avoid further long-term complications and re-operations. Though 30-day mortality and postoperative comorbidity for AAR are comparable to those in TAAR after treatment of AADA in our analysis, decisionmaking for the surgical strategy should weigh the operative risk of TAAR against the long-term outcome.

Keywords: Acute type A aortic dissection (AADA); total aortic arch replacement (TAAR); ascending aorta replacement (AAR)

Submitted Feb 13, 2020. Accepted for publication Jul 31, 2020.

doi: $10.21037 /$ jtd-20-871

View this article at: http://dx.doi.org/10.21037/jtd-20-871

(c) Journal of Thoracic Disease. All rights reserved. 


\section{Introduction}

According to the German registry of type A aortic dissection (GERAADA) the incidence of acute aortic dissection type A (AADA) ranges from 2 to 4 cases per 100,000 persons/year and correlates to 1,600 to 3,200 cases annually (1). Without emergency treatment AADA is widely known as a lifethreatening disease with a high mortality rate of $1 \%$ per hour, an expected mortality rate of over $50 \%$ during the first 3 days and reaching $80 \%$ by the third week (2). The incidence of $\mathrm{AADA}$ is increasing due to increasing average population age (3).

The therapy of choice usually consists of replacement of that part of the ascending aorta, which has a tear in the endothelial layer to restore the diseased part of the ascending aorta. In previous studies mortality rates reflect the critical state of the patients and range from $4.2 \%$ to $28.6 \%$ with variations due to the surgeon's experience and center volume (3). However, recently results have improved due to better surgical techniques and modern intensive care units (4). The German registry, one of the worldwide largest registries, reported an average 30-day mortality of $18.5 \%$ in 2017 (1). In approximately half of the AADA patients, the aortic arch is diseased, and many of those patients are postoperatively far from being cured. Regardless of the surgical techniques used, diseased aortic tissue is left in situ, which might be a source of late complications. The most susceptible region with regard to down-streaming postsurgical endothelial tears is the aortic arch, and to a slightly lower extent the descending part of the thoracic aorta. Up to $40 \%$ of patients demonstrate persistent dissection in a previously not replaced part of the aorta and in consequence, $10 \%$ to $27 \%$ of patients experience a late size increase of the false lumen, which may be a reason for late re-operation with again high perioperative mortality and morbidity (5). Therefore, it is still under debate, as whether to perform a standard hemiarch resection, where the patient survives the acute disease or to perform an extended aortic repair, in order to repair the complete aorta and improve long-term patient outcome, even if the aortic arch has no further tear.

The extension of AADA management using total arch replacement in addition to the frozen elephant trunk (FET) procedure in the emergent surgery is on one hand associated with substantial perioperative risk, such as spinal cord and intestinal ischemia but on the other hand with a proven postoperative and long-term benefit in experienced cardiac centres (6). Therefore, our study intended to compare these treatment strategies regarding the primary aspect of postoperative survival and secondarily to the neurological outcome and postoperative morbidity. We present the following article in accordance with the STROBE reporting checklist (available at http://dx.doi.org/10.21037/jtd-20871).

\section{Methods}

\section{Patient population}

This retrospective analysis included 339 consecutive patients who underwent surgery for AADA in moderate hypothermic circulatory arrest (MHCA) $\left(21-28{ }^{\circ} \mathrm{C}\right)$ from January 2001 until December 2016. Patients were divided into two groups: 47 patients underwent total aortic arch replacement (TAAR) (13.9\%) vs. 292 (86.1\%) patients who underwent ascending aorta replacement (AAR). We performed a propensity-score analysis comparing matched TAAR and AAR subgroups with 43 patients for each group.

The primary endpoint was 30-day mortality. Secondary endpoints were long-term survival, intraoperative variables, and postoperative outcomes such as redo-surgery, blood loss, ventilation time, acute renal failure and neurologic complications.

Data were supplied from the institution's database and medical records. Follow up in terms of survival was determined by inquiries at the residents' registration offices. Follow up completeness was $93 \%$, $7 \%$ were lost to follow up. The study conformed to the provisions of the Declaration of Helsinki (as revised in 2013). The study protocol was approved by the local Ethics Committee of the medical faculty of the Christian-Albrechts-Universität zu Kiel (D417/17) and informed consent was taken from the patients.

\section{Patient management}

Patients with acute type A aortic dissections were operated on an emergency basis and transported directly to the operating room. The diagnosis was confirmed preoperatively by an electrocardiography (ECG) gated contrast enhanced computed tomography (CT) to detect the exact location and extension of the dissection membrane. Patients with confirmed AADA were directly transferred to the operation room and the surgical procedure was performed on an emergency basis. If not intubated, patients were investigated for neurological symptoms and 
malperfusion signs preoperatively by physical examination. Any findings were documented on the admission sheet for further use. Transoesophageal echocardiography (TEE) was performed intraoperatively under general anaesthesia.

\section{Surgical procedure}

All patients underwent surgery by a senior surgeon. A standard median sternotomy followed by longitudinal pericardiotomy was performed under general anesthesia. Femoral retrograde and direct aortic antegrade access sites were used to establish cardiopulmonary bypass (CPB). Since 2010, CPB was established using transatrial left ventricular antegrade pulmonary vein cannulation as an alternative for arterial cannulation under moderate hypothermic circulatory arrest (MHCA) with a core temperature between 22-24 ${ }^{\circ} \mathrm{C}(7)$. Venous drainage was performed either through direct cannulation of the right atrium or through the femoral vein with a cannula that extends to the right atrium. The extent of femoral vein cannula was controlled with TEE. After cross-clamping of the aorta, a standard retrograde injection of cold Buckberg's blood cardioplegic solution for myocardial protection was performed in all cases in combination with antegrade administration through the ostia. The ascending aorta was opened and antegrade cerebral perfusion with cold oxygenated blood $\left(22-28^{\circ} \mathrm{C}\right)$ was introduced through a balloon catheter inserted into the arch vessels with flow pressure control of about 50-60 mmHg. Following our center's standard operating procedures, the distal extent of aortic repair was dependent on the extent of the dissected intimal tear. The aortic reconstruction was limited to the ascending aorta just proximal to the innominate artery if the intimal tear did not extend to or originate in the aortic arch.

Otherwise, the aortic repair was extended to a hemiarch or total arch replacement with re-implantation of the head and neck arteries by an island technique. In several cases, a FET was introduced in the proximal descending aorta. Before performing the anastomosis, the intimal tears at the proximal and distal aortic stumps were repaired using Gelatin-resorcinol-formaldehyde biologic glue, and the edges were compressed by flattened so-called Borst clamps circularly for 5 minutes. The stumps were then sharply and circularly cut to achieve a clean edge. Cerebral perfusion was removed shortly before the end of suturing of the distal anastomosis. After insertion of the perfusion cannula directly into the graft, $\mathrm{CPB}$ was slowly restarted again after careful de-airing. The proximal aortic repair was performed either through isolated supra-coronary AAR or through a replacement or reconstruction of the aortic root with an isolated aortic valve replacement. In the case of associated isolated aortic valve disease, an additional valve replacement was performed when the aorta was only reconstructed. After removing the aortic clamp, continuous $\mathrm{CO}_{2}$ insufflation was stopped. Transesophageal echocardiography was performed to control the presence of residual air in the left side of the heart. During rewarming, concomitant procedures, if required, were performed.

\section{Statistical analysis}

Statistical analysis was performed using IBM SPSS Statistics for Windows (Version 18.0). Normality of continuous variables was assessed by the Kolmogorow-Smirnov test. Normally distributed data are presented as mean \pm standard deviation and compared by unpaired $t$-test, whereas notnormally distributed continuous data are presented as medians with ranges or interquartile ranges as appropriate, and compared by the Mann-Whitney $\mathrm{U}$ test. Categorical variables are displayed as frequency distributions (n) and simple percentages (\%). Univariate comparison between the groups for categorical variables was made using the Chi2-test and the Fisher's exact test. Missing data were excluded pairwise. Statistical significance was considered when $\mathrm{P} \leq 0.05$. Variables associated with 30 -day mortality were selected due to clinical relevance and included into multivariable logistic regression analysis with backward elimination to determine their relative impact (adjusted odds ratio, OR) on 30-day mortality. Included variables were age $>75$ years, Euro-SCORE II, coronary heart disease, TAAR vs. AAR, length of surgery ( $\mathrm{min}$ ), CPB time (min), number of red blood cell concentrates intraoperatively, postoperative new-onset of hemodialysis, postoperative TIA or stroke.

Since preoperative findings were not homogeneous between the two surgical groups, statistical matching based on the propensity score was conducted to analyze survival of patients operated on by TAAR and AAR with comparable baseline characteristics. Propensity scores were calculated using multivariable logistic regression analysis with TAAR $v s$. AAR as a dependent variable and age (y), sex, arterial hypertension, chronic obstructive pulmonary disease, coronary heart disease, atrial fibrillation, previous thoracic surgery, diameter of ascending aorta prostheses $(\mathrm{mm})$, urgent or emergency admission, smoking and aortic valve vitium as independent variables. Matching was conducted pairwise 
with a maximum caliper width of 0.2 of the pooled standard deviation of the logit of the propensity score according to Austin 2011 (8). Finally, 43 patients operated on by TAAR and 43 patients by AAR were matched and their main pre-, intra and postoperative findings were summarized in Tables 1-3. Survival during postoperative follow-up was estimated by Kaplan-Meier curves for the matched and unmatched groups and compared by log-rank test (9).

\section{Results}

By comparing demographic data between both groups, it was found that patients were significantly older in the AAR group than in the TAAR group [66.0 (55.9;73.5 y) vs. 57.9 (53.6;68.3 y); $\mathrm{P}=0.049)]$. Females underwent AAR more frequently than TAAR (37.3 \% vs. $23.4 \%, \mathrm{P}=0.064)$. The EuroSCORE II was similar between both groups. $72.6 \%$ of the study population presented with arterial hypertension, followed by the presence of aortic valve insufficiency in $39.7 \%$ of patients, which occurred significantly more frequent in the AAR group than in the TAAR group (43.2\% vs. $17.5 \% ; \mathrm{P}=0.002)$. Patients with a prior aortic aneurysm represented $29.6 \%$ of the study population. Marfansyndrome was present in $2.1 \%$ of patients. $9.0 \%$ of patients were cardiopulmonary resuscitated. Cardiogenic shock was diagnosed in $7.5 \%$ of patients, and $10.1 \%$ were intubated before admission to the operating room.

Presence of coronary heart disease as well as risk factors for AADA such as arterial hypertension and aneurysms showed no significant difference between both groups. Patients who underwent TAAR were more often smokers compared to AAR $(\mathrm{P}=0.025)$. Presence of previous thoracic surgery was significantly higher in the TAAR group than in the AAR group [12 (25.5\%) vs. 24 (8.4\%); $\mathrm{P}<0.001$ ] (Table 4).

Intraoperatively, duration of surgery was significantly longer in the TAAR group than in the AAR group [363 $(280 ; 432)$ vs. 266 (220;317); $\mathrm{P}<0.001]$. Consequently, patients who underwent TAAR had significantly longer CPB times [250 (181;304) vs. $157(130 ; 196) ; \mathrm{P}<0.001]$ as well as significantly longer hypothermic circulatory arrest time [81 (49;115) vs. 31 (25;41); $\mathrm{P}<0.001]$. The number of transfused red blood cells was higher in the TAAR-group than in the AAR-group [6 (0;9) vs. $3(0 ; 6) ; \mathrm{P}=0.070]$, but however in failed significance. The surgical procedure was extended to include aortic valve replacement more frequently in the TAAR group than in the AAR group (21.3\% vs. 8.9\%; $\mathrm{P}=0.011)$. Aortic root replacement was performed in $27.7 \%$ in the TAAR vs. $10.3 \%$ in the AAR population ( $\mathrm{P}=0.001)$. David reconstruction was performed in $2.1 \%$ in the TAAR vs. $4.5 \%$ in the AAR group. The FET implantation was performed in $14.9 \%$ of patients within the TAAR group (Table 5).

Postoperatively, the incidence of acute kidney injury (AKI) and the new onset of dialysis did not differ between AAR and TAAR. The difference between clinically proven and CT-Scan controlled postoperative neurologic damage was not significantly different between both groups $(\mathrm{P}=0.522)$. The overall 30 -day mortality-rate was $17.7 \%$ $(\mathrm{n}=60)$ but did not reach significance between the two groups. $49(16.8 \%)$ patients died in the AAR group and 11 patients $(23.4 \%)$ in the TAAR group $(\mathrm{P}=0.270)$ (Table 6).

After propensity score matching, preoperative details were comparable without statistically significant differences (Table 1). However, some preoperative findings showed disparities, e.g., neurological deficits $(30.2 \%$ in the AAR group $v s .18 .6 \%$ in the TAAR group, $\mathrm{P}=0.209$ ), but failed significance.

Intraoperatively, duration of surgery was significantly longer in the matched TAAR subgroup compared to the AAR group $(365 \pm 101.9$ vs. $261 \pm 86.6 ; \mathrm{P}<0.001)$, as well as CPB times $(258 \pm 87.6$ vs. $148 \pm 47.2 ; \mathrm{P}<0.001)$ and hypothermic circulatory arrest time $[86(55 ; 128)$ vs. 28 $(22 ; 36) ; \mathrm{P}<0.001]$. The surgical procedure of TAAR includes aortic valve replacement (20.9\%). Aortic root replacement was performed in $27.9 \%$ in the matched TAAR vs. $7.0 \%$ in the AAR population $(\mathrm{P}=0.011)$. David reconstruction was performed in $2.3 \%$ of patients in the matched TAAR $v$ s. $4.7 \%$ in the AAR group. Within the TAAR group 14\% of patients received FET implantation (Table 2).

Postoperatively, similar to the unmatched groups, the incidence of $\mathrm{AKI}$ and the new onset of dialysis $(\mathrm{P}=0.955$ and 0.750 , respectively) showed no difference between AAR ad TAAR. Moreover, postoperative neurologic damage occurred with a similar frequency in both groups $(\mathrm{P}=0.323)$. However, $34.9 \%$ of TAAR-patients were reintubated in comparison to $4.7 \%$ of AAR-patients $(\mathrm{P}<0.001)$. The 30-day-mortality showed no statistically significant difference between the matched AAR and TAAR group $(\mathrm{P}=0.436)$ (Table 3). Figure 1 shows the 30 -day-mortality of patients treated by AAR compared to TAAR for the original groups and the matched subgroups.

The survival curves are presented in Figures 2 and 3 and showed no statistical difference between the unmatched TAAR and the AAR groups $(\mathrm{P}=0.436)$ as well as between the matched subgroups $(\mathrm{P}=0.503)$.

In the univariate analysis the DeBakey Classification 
Table 1 Demographic and clinical characteristics of propensity-matched patient subgroups

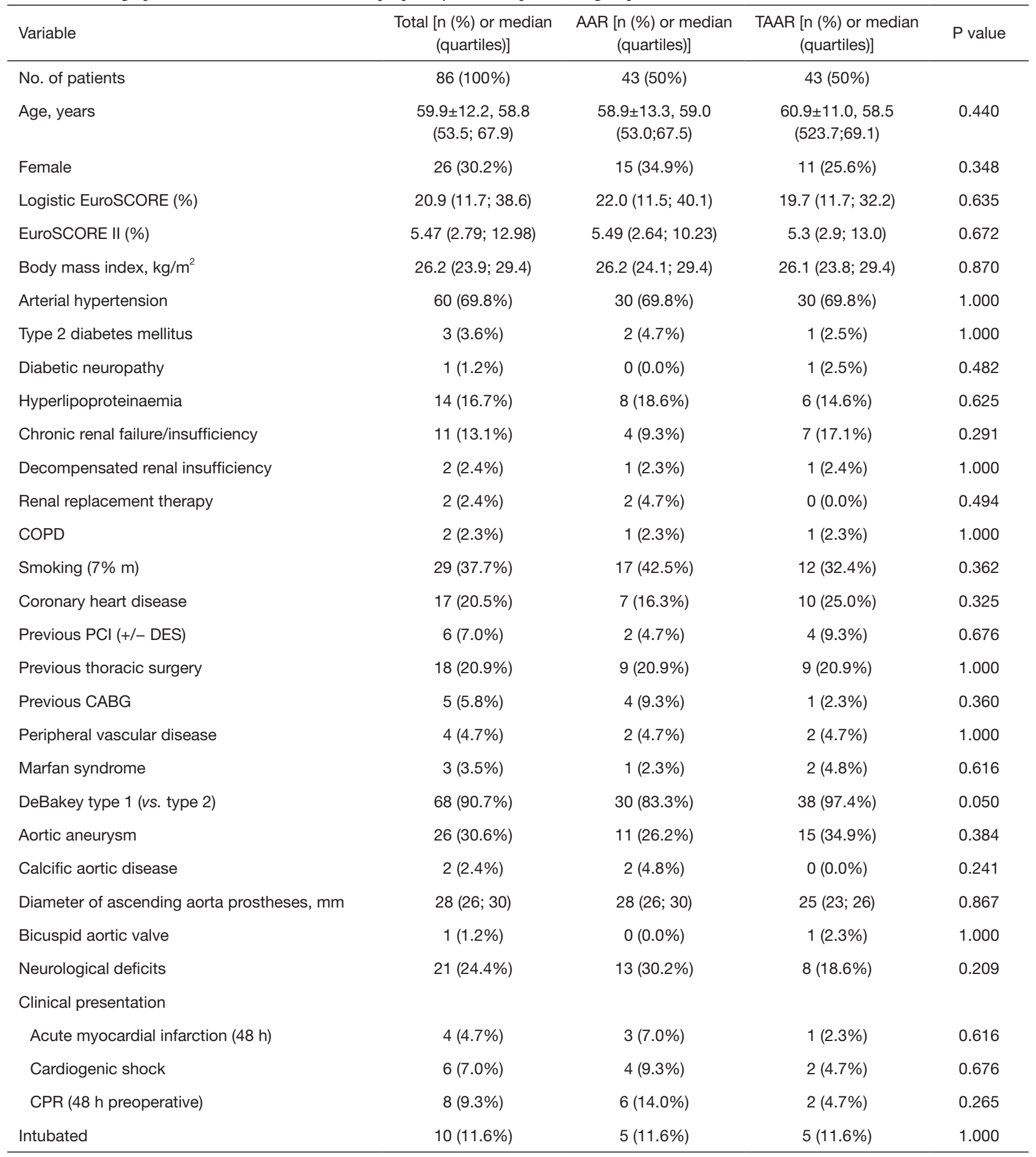

EuroSCORE, European System for Cardiac Operative Risk Evaluation; COPD, chronic obstructive pulmonary disease; PCI, percutaneous coronary intervention; DES, drug eluting stent; CABG, coronary artery bypass grafting; CPR, cardiopulmonary resuscitation; $\mathrm{m}$, missing values $>5 \%$. 
Table 2 Operative data of matched patient subgroups

\begin{tabular}{|c|c|c|c|c|}
\hline Variable & $\begin{array}{l}\text { Total }[\mathrm{n}(\%) \text { or median } \\
\text { (quartiles)] }\end{array}$ & $\begin{array}{c}\text { AAR }[\mathrm{n}(\%) \text { or median } \\
\text { (quartiles)] }\end{array}$ & $\begin{array}{c}\text { TAAR }[\mathrm{n}(\%) \text { or median } \\
\text { (quartiles)] }\end{array}$ & $P$ value \\
\hline Length of surgery, min & $313 \pm 107.6$ & $261 \pm 86.6$ & $365 \pm 101.9$ & $<0.001$ \\
\hline Cardiopulmonary bypass time, $\min$ & $203 \pm 89.2$ & $148 \pm 47.2$ & $258 \pm 87.6$ & $<0.001$ \\
\hline Cross-clamp time, min & $96(70 ; 153)$ & $73(52 ; 96)$ & $139(100 ; 209)$ & $<0.001$ \\
\hline Number of fresh frozen plasma cells & $0(0 ; 6)$ & $0(0 ; 6)$ & $0(0 ; 5)$ & 0.760 \\
\hline Number of platelets concentrate & $2(1 ; 2)$ & $2(1 ; 2)$ & $2(2 ; 2)$ & 0.248 \\
\hline \multicolumn{5}{|l|}{ Surgical procedure } \\
\hline CABG & $7(8.1 \%)$ & $4(9.3 \%)$ & $3(7.0 \%)$ & 1.000 \\
\hline Aortic valve replacement & $9(10.5 \%)$ & $0(0.0 \%)$ & $9(20.9 \%)$ & 0.002 \\
\hline Mitral valve reconstruction/replacement & $1(1.2 \%)$ & $0(0.0 \%)$ & $1(2.3 \%)$ & 1.000 \\
\hline TEVAR (EVAR) & $7(8.1 \%)$ & $1(2.3 \%)$ & $6(14.0 \%)$ & 0.110 \\
\hline \multicolumn{5}{|l|}{ Arterial cannulation $(14 \% m)$} \\
\hline Femoral artery & $10(13.2 \%)$ & $9(24.3 \%)$ & $1(2.6 \%)$ & \\
\hline Ascending aorta & $20(26.3 \%)$ & $9(24.3 \%)$ & $11(28.2 \%)$ & \\
\hline Right atrium & $71(93.4 \%)$ & 34 (91.9\%) & 37 (94.9\%) & \\
\hline Bicaval & $3(3.9 \%)$ & $2(5.4 \%)$ & $1(2.6 \%)$ & \\
\hline Femoral vein & $2(2.6 \%)$ & $1(2.7 \%)$ & $1(2.6 \%)$ & \\
\hline
\end{tabular}

CABG, coronary artery bypass grafting; TEVAR, thoracic endovascular aortic repair; EVAR, endovascular aortic repair; m, missing values $>5 \%$.

Type I and II was not associated with 30-day $(\mathrm{P}=0.78)$ and long-term $(\mathrm{P}=0.76)$ mortality in both groups. Multivariable analysis identified the EuroSCORE II, long operation time, the number of intraoperatively administered red blood cell concentrates, postoperative dialysis and postoperative TIA or stroke as independent risk factors for 30-day mortality. However, TAAR and AAR itself were no predictors for 30-d-mortality in this study (Table 7).

\section{Discussion}

In this retrospective study we demonstrate that the overall postoperative 30-day mortality-rate for all patients suffering from acute Type A dissection was $17.7 \%(n=60 / 339$ patients). No significant difference $(\mathrm{P}=0.27)$ was seen between the AAR (16.8\%) and the TAAR group (23.4\%). In contrast, the operative time, $\mathrm{CPB}$, cross-clamp and ischemic time were significantly longer in the TAAR group $(\mathrm{P}<0.001)$ 
Table 3 Postoperative data and outcomes of matched patient subgroups

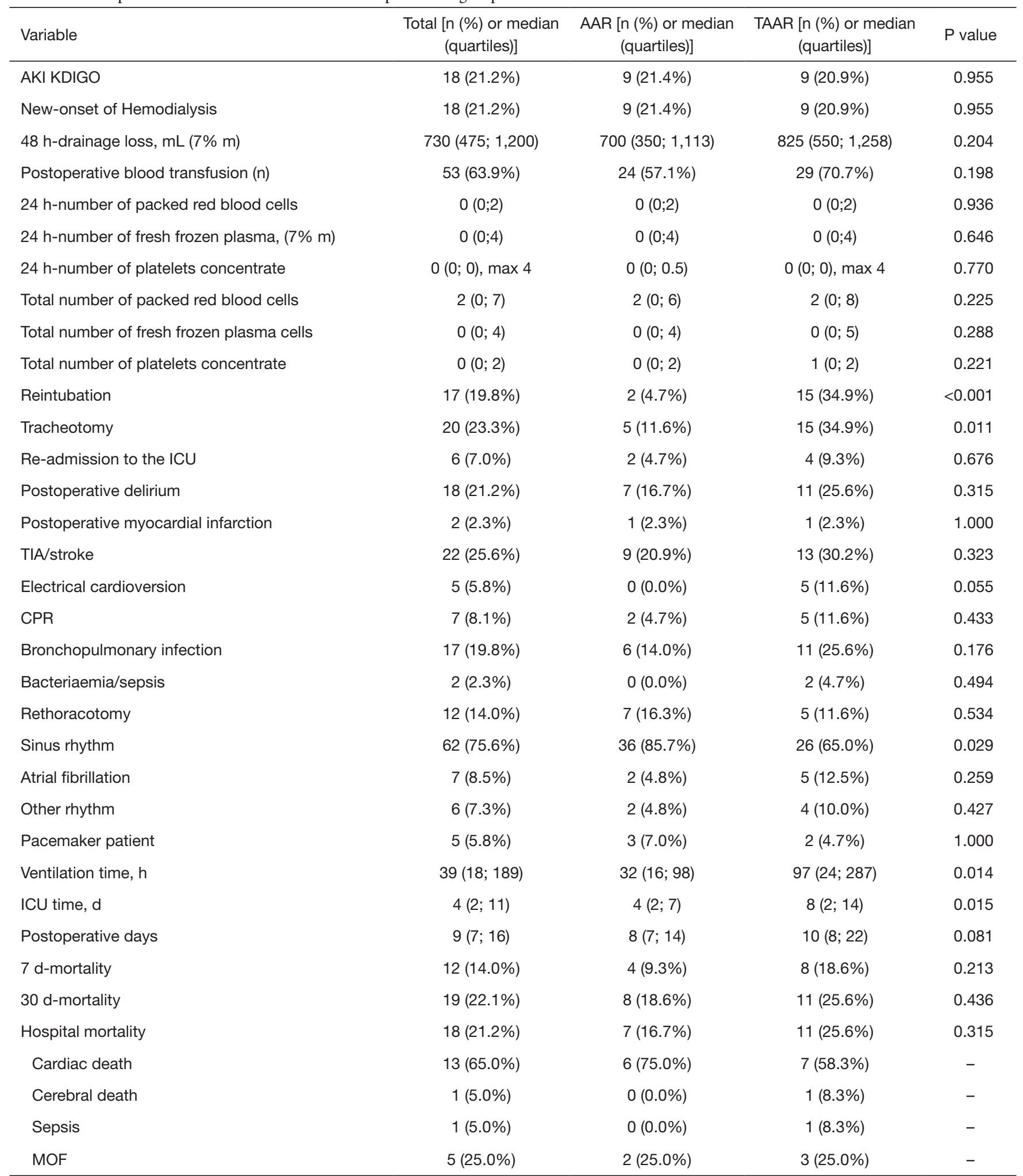

AKI, acute kidney insufficiency; KDIGO, Kidney Disease, Improving Global Outcomes; IABP, intraaortic balloon pump; ECLS, extracorporeal live support; TIA, transient ischemic attack; CPR, cardiopulmonary resuscitation; VAC, vacuum assisted closure-therapy; ICU, intensive care unit; POD, postoperative days; MOF, multiorgan failure; $m$, missing values $>5 \%$. 
Table 4 Demographic and clinical characteristics of the study population

\begin{tabular}{|c|c|c|c|c|}
\hline Characteristics & $\begin{array}{l}\text { Total }[\mathrm{n}(\%) \text { or median } \\
\text { (quartiles)] }\end{array}$ & $\begin{array}{c}\text { AAR }[\mathrm{n}(\%) \text { or median } \\
\text { (quartiles)] }\end{array}$ & $\begin{array}{c}\text { TAAR [n (\%) or median } \\
\text { (quartiles)] }\end{array}$ & $P$ value \\
\hline No. of patients 339 & $339(100 \%)$ & $292(86.1 \%)$ & $47(13.9 \%)$ & \\
\hline Age, years & $\begin{array}{c}63.7 \pm 12.1,65.6 \\
(55.1 ; 72.8)\end{array}$ & $\begin{array}{l}64.2 \pm 12.2,66.0 \\
(55.9 ; 73.5)\end{array}$ & $\begin{array}{c}60.5 \pm 10.7,57.9 \\
(53.6 ; 68.3)\end{array}$ & 0.049 \\
\hline Age $\geq 70$ years & $124(36.6 \%)$ & $114(39.0 \%)$ & $10(21.3 \%)$ & 0.019 \\
\hline Logistic EuroSCORE (\%) & $23.7(12.2 ; 41.5)$ & $25.5(12.7 ; 41.7)$ & $19.7(11.7 ; 32.2)$ & 0.124 \\
\hline EuroSCORE II (\%) & $5.46(2.96 ; 12.52)$ & $5.45(2.89 ; 12.28)$ & $5.8(2.9 ; 13.4)$ & 0.851 \\
\hline Body mass index, $\mathrm{kg} / \mathrm{m}^{2}$ & $26.3(23.9 ; 29.1)$ & $26.3(23.9 ; 29.1)$ & $26.4(23.9 ; 29.3)$ & 0.933 \\
\hline Body mass index $>30\left(\mathrm{~kg} / \mathrm{m}^{2}\right)$ & $69(20.4 \%)$ & $60(20.6 \%)$ & $9(19.1 \%)$ & 0.817 \\
\hline Type 2 diabetes mellitus & $20(6.2 \%)$ & $19(6.8 \%)$ & $1(2.3 \%)$ & 0.495 \\
\hline Diabetic neuropathy & $1(0.3 \%)$ & $0(0.0 \%)$ & $1(2.3 \%)$ & 0.136 \\
\hline Hyperlipoproteinemia & $47(14.6 \%)$ & $39(14.1 \%)$ & $8(17.8 \%)$ & 0.515 \\
\hline Chronic renal failure/insufficiency & $47(14.5 \%)$ & $40(14.3 \%)$ & $7(15.6 \%)$ & 0.822 \\
\hline Decompensated renal insufficiency & $9(2.8 \%)$ & $8(2.9 \%)$ & $1(2.2 \%)$ & 1.000 \\
\hline Renal replacement therapy & $7(2.1 \%)$ & $7(2.5 \%)$ & $0(0.0 \%)$ & 0.599 \\
\hline COPD & $21(6.4 \%)$ & $20(7.1 \%)$ & $1(2.1 \%)$ & 0.332 \\
\hline Peripheral vascular disease & $16(4.9 \%)$ & $14(5.0 \%)$ & $2(4.3 \%)$ & 1.000 \\
\hline Marfan syndrome & $7(2.1 \%)$ & $5(1.8 \%)$ & $2(4.3 \%)$ & 0.252 \\
\hline DeBakey type 1 (vs. type 2) & $240(79.7 \%)$ & $199(76.8 \%)$ & $41(97.6 \%)$ & 0.002 \\
\hline Aortic aneurysm & $100(29.6 \%)$ & $83(28.5 \%)$ & $17(36.2 \%)$ & 0.286 \\
\hline Diameter of aneurysm, mm (24\% m) & $52 \pm 10$ & $51 \pm 10$ & $57 \pm 12$ & 0.092 \\
\hline Calcific aortic disease & $8(2.4 \%)$ & $8(2.8 \%)$ & $0(0.0 \%)$ & 0.606 \\
\hline Diameter of ascending aorta prostheses, $\mathrm{mm}$ & $28(28 ; 30)$ & $30(28 ; 30)$ & $28(26 ; 30)$ & 0.001 \\
\hline Bicuspid aortic valve & $7(2.1 \%)$ & $6(2.1 \%)$ & $1(2.1 \%)$ & 1.000 \\
\hline Aortic valve insufficiency & $115(39.7 \%)$ & $108(43.2 \%)$ & $7(17.5 \%)$ & 0.002 \\
\hline Neurological deficits & $69(20.8 \%)$ & $61(21.5 \%)$ & $8(17.0 \%)$ & 0.486 \\
\hline \multicolumn{5}{|l|}{ Clinical presentation } \\
\hline Acute myocardial infarction (48 h) & $14(4.2 \%)$ & $12(4.2 \%)$ & $2(4.3 \%)$ & 1.000 \\
\hline
\end{tabular}

Table 4 (continued) 
Table 4 (continued)

\begin{tabular}{|c|c|c|c|c|}
\hline Characteristics & $\begin{array}{c}\text { Total [n (\%) or median } \\
\text { (quartiles)] }\end{array}$ & $\begin{array}{c}\text { AAR }[\mathrm{n}(\%) \text { or median } \\
\text { (quartiles)] }\end{array}$ & $\begin{array}{c}\text { TAAR [n (\%) or median } \\
\text { (quartiles)] }\end{array}$ & $P$ value \\
\hline Cardiogenic shock & $25(7.5 \%)$ & $22(7.7 \%)$ & $3(6.4 \%)$ & 1.000 \\
\hline CPR (48 h) & $30(9.0 \%)$ & $28(9.7 \%)$ & $2(4.3 \%)$ & 0.282 \\
\hline Intubated & $34(10.1 \%)$ & $28(9.7 \%)$ & $6(12.8 \%)$ & 0.601 \\
\hline
\end{tabular}

EuroSCORE, European System for Cardiac Operative Risk Evaluation; COPD, chronic obstructive pulmonary disease; PCI, percutaneous coronary intervention; DES, drug eluting stent; CABG, coronary artery bypass grafting; CPR, cardiopulmonary resuscitation; m, missing values $>5 \%$.

Table 5 Operative data

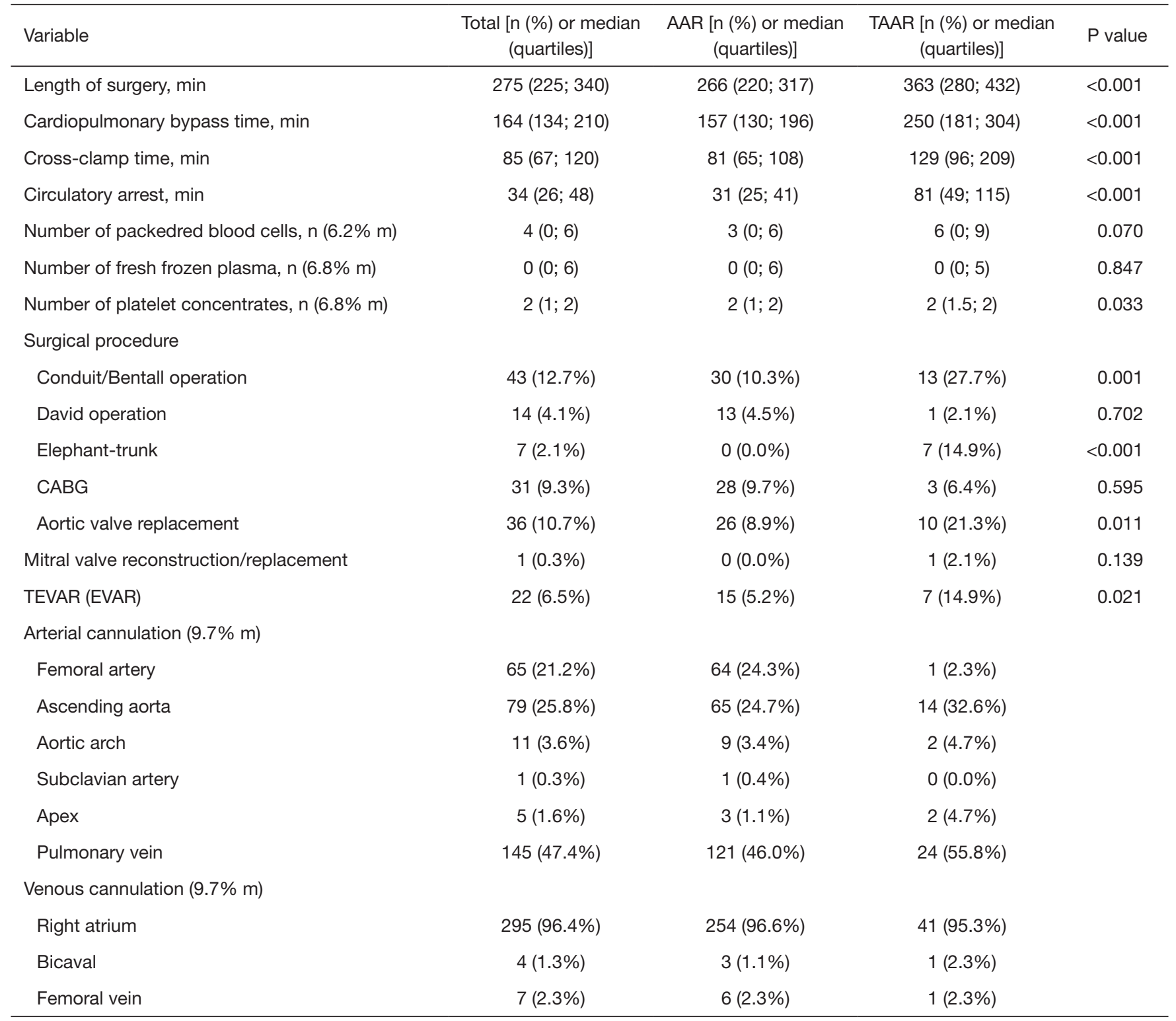

CABG, coronary artery bypass grafting; TEVAR, thoracic endovascular aortic repair; EVAR, endovascular aortic repair; m, missing values $>5 \%$. 
Table 6 Postoperative data and outcomes

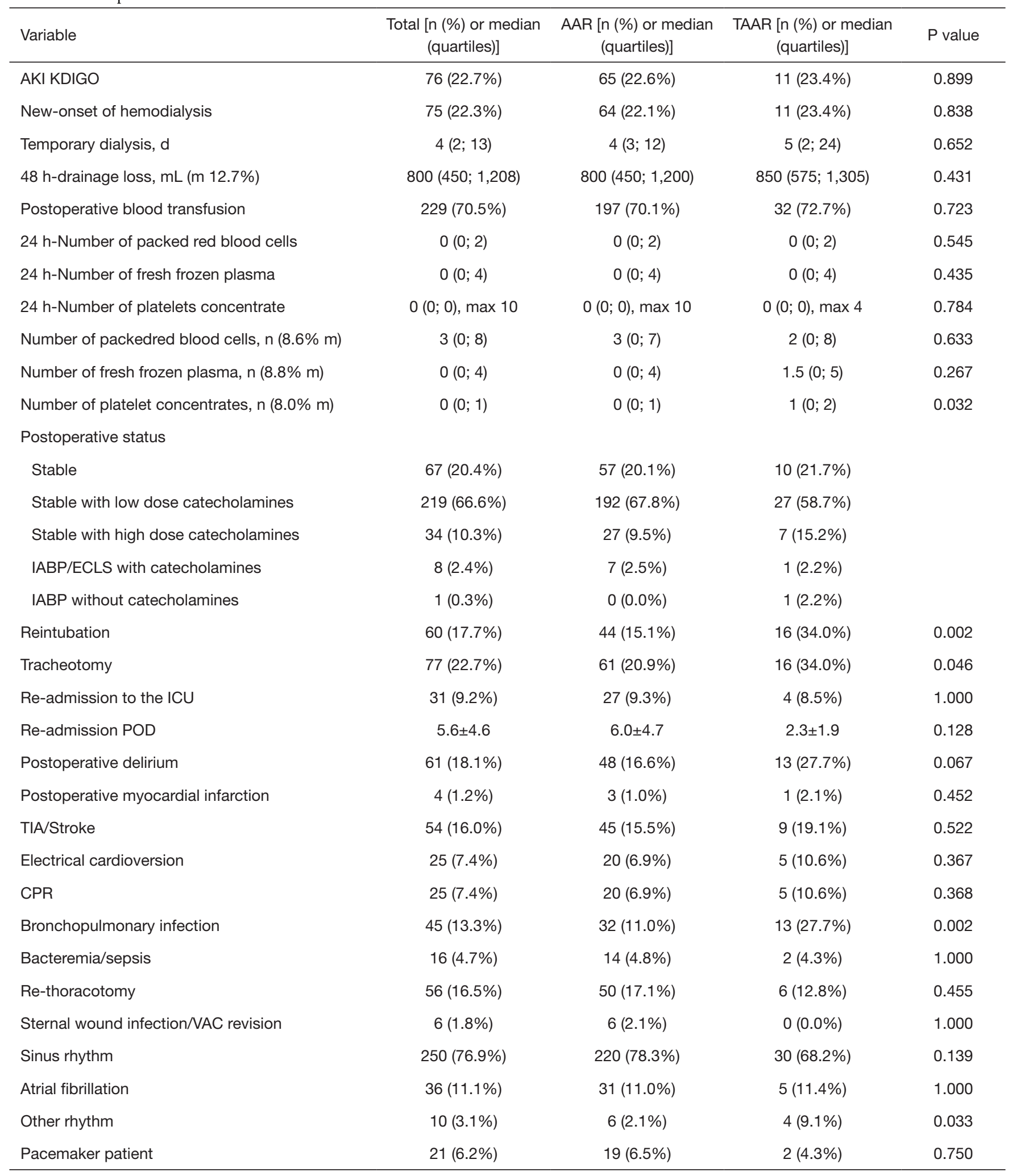

Table 6 (continued) 
Table 6 (continued)

\begin{tabular}{|c|c|c|c|c|}
\hline Variable & $\begin{array}{c}\text { Total }[\mathrm{n}(\%) \text { or median } \\
\text { (quartiles)] }\end{array}$ & $\begin{array}{c}\text { AAR [n (\%) or median } \\
\text { (quartiles)] }\end{array}$ & $\begin{array}{c}\text { TAAR [n (\%) or median } \\
\text { (quartiles)] }\end{array}$ & $P$ value \\
\hline Ventilation time, $\mathrm{h}$ & $70(21 ; 189)$ & $64(21 ; 173)$ & $129(25 ; 287)$ & 0.087 \\
\hline Postoperative days & $11(7 ; 19)$ & $11(7 ; 18)$ & $11(8 ; 22)$ & 0.634 \\
\hline Hospital mortality & $57(17.3 \%)$ & $46(16.3 \%)$ & $11(23.4 \%)$ & 0.230 \\
\hline Cardiac death & 32 (53.3\%) & $25(52.1 \%)$ & 7 (58.3\%) & \\
\hline Cerebral death & $4(6.7 \%)$ & $3(6.3 \%)$ & $1(8.3 \%)$ & \\
\hline
\end{tabular}

AKI, acute kidney insufficiency; KDIGO, Kidney Disease, Improving Global Outcomes; IABP, intraaortic balloon pump; ECLS, extracorporeal live support; TIA, transient ischemic attack; CPR, cardiopulmonary resuscitation; VAC, vacuum assisted closure-therapy; ICU, intensive care unit; $\mathrm{POD}$, postoperative days; $M O F$, multiorgan failure; $m$, missing values $>5 \%$.

Table 7 Multivariable analysis on risk factors for 30-d-mortality

\begin{tabular}{lccc}
\hline Variable & $P$ & Odds ratio & Confidence interval \\
\hline EuroSCORE II & 0.004 & 1.046 & $1.014-1.079$ \\
Length of surgery, min & 0.016 & 1.005 & $1.001-1.009$ \\
RBC intraoperative, $\mathrm{n}$ & 0.002 & 1.156 & $1.052-1.269$ \\
New-onset of hemodialysis & $<0.001$ & 5.573 & $2.522-12.313$ \\
Postoperative TIA or stroke & 0.016 & 3.195 & $1.246-8.194$ \\
\hline
\end{tabular}

EuroSCORE, European System for Cardiac Operative Risk Evaluation; RBC, red blood cell concentrates; TIA, transient ischemic attack.

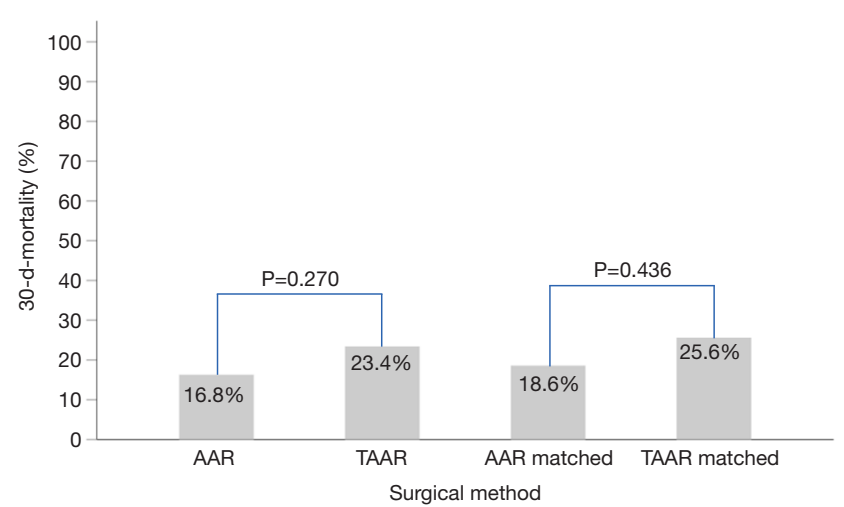

Figure 1 Showing 30-day-mortality in the matched groups and the unmatched subgroups.

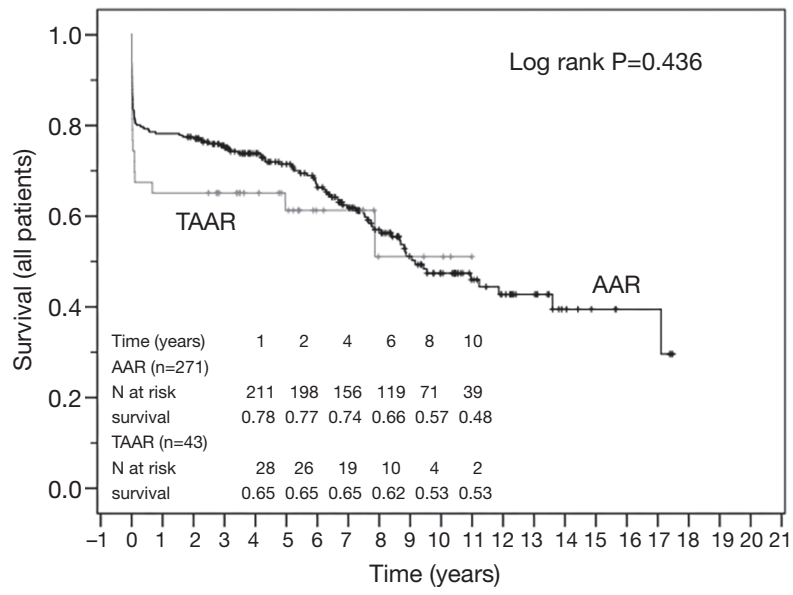

Figure 2 Kaplan-Meier curves of the original groups. 


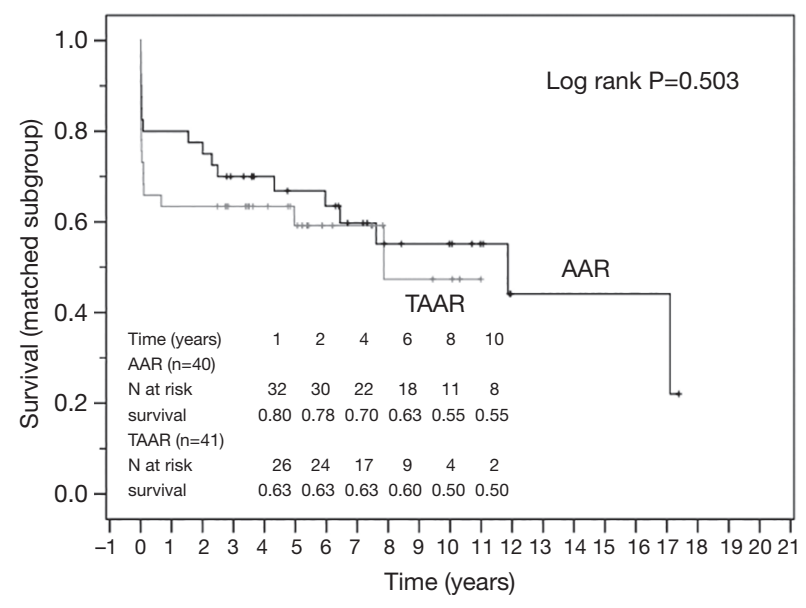

Figure 3 Kaplan-Meier curves of the matched subgroups.

than in the AAR group. However, after propensity-score matching no difference in the 30-day-mortality was seen between the subgroups $(\mathrm{P}=0.44)$.

Multivariable analysis in this study identified the EuroSCORE II, long operation time, the number of intraoperatively administered packed red blood cells, postoperative dialysis, and postoperative TIA or stroke as independent risk factors for the 30-day mortality. In the univariate analysis the aortic dissection DeBakey Classification Type I and II was not associated with 30-day $(\mathrm{P}=0.78)$ and long-term mortality $(\mathrm{P}=0.76)$ in both study groups.

In general, acute aortic dissection is a life-threatening condition that invariably requires emergency surgical intervention. The operative concept of resecting the tear from the ascending aorta is internationally accepted and is daily clinical practice worldwide. Nevertheless, it is still under debate as to whether to leave further tears in the arch, the distal arch and the descending aorta untreated, or to expand the surgical strategy and to treat the arch and the proximal descending aorta for preventive reasons only.

Our study underlines the fact that AAR and TAAR showed no significant difference in postoperative outcome according to 30 -day mortality $(\mathrm{P}=0.27)$, even though TAAR is associated with longer cross-clamping and operation time $(\mathrm{P}=0.035)$, which predicts co-morbidity and worse early outcome after surgery.

The German Registry for Acute Aortic Dissection Type A analyzed the effect of different operative strategies with respect to the treatment of the aortic arch with regard to postoperative mortality and new onset of neurological adverse outcomes, malperfusion as well as mortality: the overall mortality was up to $20.2 \%$ and was higher than in our study population. The mortality in their AAR group tended to be lower than in their TAAR-group $(18.7 \% v s$. $25.7 \%)$, although without a significant statistical difference $(\mathrm{P}=0.07)(10)$.

The interesting results of the German Registry are confirmed by this study: the extent of the dissection classified by DeBakey had no influence on the short $(\mathrm{P}=0.76)$ and long-term outcome $(\mathrm{P}=0.78)$. Depending on the extent of the disease, a total resection of all the teared aorta as in this study described. Therefore, a patient-based treatment plan in case of type A dissection depending on preoperative age and patient's risk factors should define whether to replace the ascending aorta alone or to replace the aortic arch for preventive and prognostic reasons.

The standard surgical treatment of AADA is known to be the emergency replacement of that part of the ascending aorta with the tear in the endothelial layer. Increasingly more extensive procedures including FET have been carried out during the last years, particularly for a more complex dissection of the aortic arch, or if the thoracic aorta is dilated or malperfusion is present in the descending part of the thoracic aorta $(11,12)$. The remaining untreated diseased tissue of the aortic arch and the descending part of the thoracic aorta are responsible for the postsurgical complications (13). The use of FET prostheses in our patient population with first-time aortic arch replacement is rare with only $2.1 \%$ (7/47patients) though many advantages are proposed. The current strategy is to implant the FET in zone I or II of the aortic arch and bypass the left subclavian artery or any supraaortal vessel even in experienced aortic centers to reduce longer operation times, which reflects the complexity of FET prosthesis implantation methods to the descending aorta $(14,15)$.

In contrast to the wide use of FET prothesis, Kobuch et al. stated that re-operation could be carried out with low mortality rate if necessary with several complications after primary surgery for type A dissection. For those patients, FET had become a favored technique in case of reoperation after initial conventional surgery for Type A dissection to restore the descending part of the aorta, to avoid further re-operation and to enable the implantation of further endovascular stents in the descending aorta if necessary (6). FET was recommended also in a study from Tsagakis et al. (14). Additionally, Luehr et al. described that $12.6 \%$ of patients had adverse events and redo surgery after a median follow-up time of 4 years due to aortic dilatation, rupture of 
the aorta and endoleaks in a retrospective multicenter study on elective aortic arch surgery (16).

Bringing all experiences together, Leone et al. reviewed 437 patients with different FET prosthesis for a mix of complex aortic diseases and showed acceptable postoperative results with a remarkable low rate of $5 \%$ paraplegia rate and $10 \%$ neurological deficiencies with overall mortality rate of $14.9 \%(17)$.

The current analysis showed an overall 30-day mortalityrate of $17.7 \%(n=60)$. The mortality rate in patients with AAR vs. TAAR did not reach statistical significance $[16.8 \%$ $(\mathrm{n}=49)$ vs. $23.4 \%(\mathrm{n}=11) ; \mathrm{P}=0.270]$. Moreover, no significant difference regarding postoperative neurologic adverse outcome between the study groups was detected $(\mathrm{P}=0.522)$.

Furthermore, we used Kaplan-Meier estimator to analyze the long-term survival. The analysis showed a satisfactory result without statistical differences between the presented groups.

Czerny et al. compared the perioperative death rates which strongly depended on the number of organs affected by malperfusion before and after AADA surgery in patients included in GERAADA. The mortality varied according to the number of organs affected by malperfusion (none, $12.6 \% ; 1$ system, $21.3 \%$; 2 systems, $30.9 \%$; 3 systems, $43.4 \% ; \mathrm{P}<0.001)(18)$. Though we couldn't find a significant influence of the extent of the aortic dissection on short and long-term mortality in our patients, organ perfusion might also have an impact on the survival. These findings could play an important role in future decisions regarding the surgical repair strategy of AADA whether to replace only the ascending aorta or to replace the arch in total with the proximal descending aorta. With the capacity to start early distal perfusion as soon as possible, the effect of the extended surgical duration on the mortality and comorbidity in TAAR could be further minimized.

Previous studies showed that increased age in general is associated with increased short- and long-term mortality rates after surgical repair of AADA independent from the extent of the performed surgical repair $(19,20)$. We found that patients were significantly older in the AAR group than in the TAAR group [66.0 vs. $57.9 \mathrm{y} ; \mathrm{P}=0.049]$. This could be attributed to the tendency of performing the less aggressive surgical repair in elderly patients. A study from Trimarchi et al. confirmed that increased age (70 years or more) is an independent predictor of in-hospital mortality [38.2\% ( $>70$ y) vs. $26.0 \%$ ( $<70$ y); $\mathrm{P}<0.001$, odds ratio 1.73] (21). Though in our study the mean age of patients in the AAR group is significantly younger, $1 / 3$ of our patients are older than 70 years in total and therefore associated with higher risk of perioperative mortality. The overall conclusion from those previous findings focused on the role of age in those patients undergoing surgery of AADA. However, the role of the extension of the surgical procedure performed including TAAR on postoperative mortality rate still remains to be clarified.

A large study from the International Registry of Acute Aortic Dissection (IRAD) reported preoperative shock conditions, aortic rupture, neurologic damage, and organ ischemia as major determinants of outcomes after surgical repair of AADA. Unstable patients had almost double the postoperative mortality rate when compared with stable patients $(31.4 \%$ vs. $16.7 \%)$. Those findings were independent of the extent of the surgical procedure $(22,23)$. According to the propensity matching in our study including 43 patients in each group of AAR and TAAR who were matched according to preoperative parameters such as age and gender of patients, coronary heart disease and arterial hypertension, we found that the mortality between both groups did not differ significantly $(\mathrm{P}=0.436)$.

The overall mortality rate in patients undergoing emergency surgery for AADA is still high. However, we concluded from this study as well as from similar studies that the more aggressive surgical repair including the total arch replacement by itself is not associated with higher mortality rates compared to other more conservative surgeries. We believe that in patients suffering from AADA the surgical decision should be taken on an individual basis according to various parameters: the preoperative condition as well as the age of the patients and the presence of multiorgan malperfusion should be the main determinants as whether to perform a more aggressive surgical repair or not. Various single-centres reported satisfactory results and fewer mortality rates between $2.8 \%$ to $9 \%$ in comparison to large registries $(24,25)$.

Our results may reflect the improvement of postoperative mortality after AADA in general. With surgeon practice, increasing experience, implementation of precise criteria for surgical decisions, as well as minimising the duration of malperfusion, we believe that the outcomes after AADA will be continuously improved.

\section{Limitations of our study}

The main limitation of this study is its retrospective design and the risk of bias due to unknown confounding variables as well as the surgeon and an individual patient depending 
decision making in a non-randomized patient population. Therefore, we conducted propensity score matching, however this statistical method cannot fully prevent confounding and the resulting small size of the matched subgroups reduces the statistical power to detect significant differences. Though we are aware of the fact, that many publications focused on the differentiation between the aorta related and non-aorta-related mortality, we believe that the overall mortality reflects additional important aspects like age and co-morbidities which are associated with the aortic disease, therefore providing a more realistic view of the long-term outcome after surgical therapy of the dissected aorta. This study is based on single-centre experience with high experienced surgeons performing the procedure.

\section{Conclusions}

Both TAAR and AAR demonstrated a comparable postsurgical outcome regarding their mortality and morbidity after surgical repair of AADA. The decision considering the extent of surgical repair of AADA should be taken on an individual basis according to the preoperative condition, age of the patients and the presence of preoperative extension of the dissection.

\section{Acknowledgments}

We are very grateful for the DZHK (German Center for Heart and Circulation) funding.

Funding: None.

\section{Footnote}

Reporting Checklist: The authors have completed the STROBE reporting checklist. Available at http://dx.doi. org/10.21037/jtd-20-871

Data Sharing Statement: Available at http://dx.doi. org/10.21037/jtd-20-871

Conflicts of Interest: All authors have completed the ICMJE uniform disclosure form (available at http://dx.doi. org/10.21037/jtd-20-871). The authors have no conflicts of interest to declare.

Ethical Statement: The authors are accountable for all aspects of the work in ensuring that questions related to the accuracy or integrity of any part of the work are appropriately investigated and resolved. The study conformed to the provisions of the Declaration of Helsinki (as revised in 2013). The study protocol was approved by the local Ethics Committee of the medical faculty of the Christian-Albrechts-Universität zu Kiel (D417/17) and informed consent was taken from the patients.

Open Access Statement: This is an Open Access article distributed in accordance with the Creative Commons Attribution-NonCommercial-NoDerivs 4.0 International License (CC BY-NC-ND 4.0), which permits the noncommercial replication and distribution of the article with the strict proviso that no changes or edits are made and the original work is properly cited (including links to both the formal publication through the relevant DOI and the license). See: https://creativecommons.org/licenses/by-nc-nd/4.0/.

\section{References}

1. Rampoldi V, Trimarchi S, Eagle KA, Isselbacher Simple risk models to predict surgical mortality in acute type A aortic dissection: The International Registry of Acute Aortic Dissection score. International Registry of Acute Aortic Dissection (IRAD) Investigators. Ann Thorac Surg 2007;83:55-61.

2. Criado FJ. Aortic dissection: a 250-year perspective. Tex Heart Inst J 2011;38:694-700.

3. Umana-Pizano JB, Nissen AP, Sandhu HK, Acute Type A Dissection Repair by High-Volume vs. Low-Volume Surgeons at a High-Volume Aortic Center. Ann Thorac Surg 2019;108:1330-6.

4. Clouse WD, Hallett JW, Schaff HV, Acute Aortic Dissection: Population-Based Incidence Compared with Degenerative Aortic Aneurysm Rupture. Mayo Clinic Proceedings 2004;79:176-80.

5. Cecconi M, Lopez E, Zanoli R, Postoperative follow-up of type A aortic dissection experience with 67 patients. G Ital Cardiol 1999;29:898-909.

6. Kobuch R, Hilker M, Rupprecht L, Late reoperations after repaired acute type A aortic dissection. J Thorac Cardiovasc Surg 2012;144:300-7.

7. Schoeneich F, Rahimi A, Eide M, et al. Transatrial left ventricular cannulation for arterial return in CABG with severe aortic calcification. Thorac Cardiovasc Surg 2012;60:60.

8. Austin PC. Optimal caliper widths for propensityscore matching when estimating differences in means 
and differences in proportions in observational studies. Pharmaceutical Statistics 2011;10:150-61.

9. Kaplan EL, Meier P. Nonparametric estimation from incomplete observations. J Amer Statist Assoc 1958;53:457-81.

10. Boening A, Karck M, Conzelmann LO, et al. German Registry for Acute Aortic Dissection Type A: Structure, Results, and Future Perspectives. Thorac Cardiovasc Surg 2017;65:77-84.

11. Fukuhara S, Roselli EE. Modified branched reverse frozen elephant trunk repair for failed TEVAR. Ann Cardiothorac Surg 2018;7:437-42.

12. Roselli EE, Bakaeen FG, Johnston DR, Role of the frozen elephant trunk procedure for chronic aortic dissection. Eur J Cardiothorac Surg 2017;51:i35-9.

13. Di Bartolomeo R, Leone A, Di Marco L, When and how to replace the aortic arch for type A dissection. Ann Cardiothorac Surg 2016;5:383-8.

14. Tsagakis K, Dohle DS, Wendt D, Left subclavian artery rerouting and selective perfusion management in frozen elephant trunk surgery. Minim Invasive Ther Allied Technol 2015;24:311-6.

15. Gottardi R, Voetsch A, Krombholz-Reindl P, Comparison of the conventional frozen elephant trunk implantation technique with a modified implantation technique in zone 1. Eur J Cardiothorac Surg 2020;57:669-75.

16. Luehr M, Peterss S, Zierer A, Aortic events and reoperations after elective arch surgery: incidence, surgical strategies and outcomes. Eur J Cardiothorac Surg 2018;53:519-24.

17. Leone A, Beckmann E, Aandreas M, Total aortic arch replacement with frozen elephant trunk technique: Results from two European institutes. J Thorac Cardiovasc Surg

Cite this article as: Salem M, Friedrich C, Rusch R, Frank D, Hoffmann G, Lutter G, Berndt R, Cremer J, Haneya A, Puehler $T$. Is total arch replacement associated with an increased risk after acute type A dissection? J Thorac Dis 2020;12(10):5517-5531. doi: $10.21037 /$ jtd-20-871
2020;159:1201-11.

18. Czerny M, Schoenhoff F, Etz C, et al. The impact of preoperative malperfusion on outcome in acute type $\mathrm{A}$ aortic dissection - results from GERAADA. J Am Coll Cardiol 2015;65:2628-35.

19. Mehta RH, O'Gara PT, Bossone E, et al. Acute type A aortic dissection in the elderly: clinical characteristics, management, and outcomes in the current era. J Am Coll Cardiol 2002;40:685-92.

20. Tan ME, Morshuis WJ, Dossche KM, Long-term results after 27 years of surgical treatment of acute type A aortic dissection. Ann Thorac Surg 2005;80:523-9.

21. Trimarchi S, Eagle KA, Nienaber CA, Role of age in acute type A aortic dissection outcome: report from the International Registry of Acute Aortic Dissection (IRAD). J Thorac Cardiovasc Surg 2010;140:784-9.

22. Tsai TT, Evangelista A, Nienaber CA, Long-term survival in patients presenting with type A acute aortic dissection: insights from the International Registry of Acute Aortic Dissection (IRAD). Circulation 2006;114:I350-6.

23. Teman NR, Peterson MD, Russo MJ, Outcomes of patients presenting with acute type A aortic dissection in the setting of prior cardiac surgery: an analysis from the International Registry of Acute Aortic Dissection. Circulation 2013;128:S180-5.

24. Sun L, Qi R, Zhu J, et al. Repair of acute type A dissection: our experiences and results. Ann Thorac Surg 2011;91:1147-52.

25. Andersen ND, Ganapathi AM, Hanna JM, et al. Outcomes of acute type A dissection repair before and after implementation of a multidisciplinary thoracic aortic surgery program. J Am Coll Cardiol 2014;63:1796-803. 